\title{
El día después del smartphone: escenarios en la evolución de dispositivos móviles
}

The day after the smartphone: scenarios in the evolution of mobile devices

Francisco Vacas Aguilar, Universidad Rey Juan Carlos - pvacas2006@gmail.com

\section{Resumen}

Un decenio después del lanzamiento del iPhone de Apple, el smartphone se ha convertido en el dispositivo básico y central de la comunicación personal y acceso a la red. No obstante, las señales de madurez que ya empiezan a aparecer en los principales mercados y el hecho de que la evolución tecnológica continua, nos indica que los smartphones serán sustituidos por algún dispositivo cuya forma y funciones aún no conocemos. En este articulo se analizan los posibles escenarios de una etapa post-smartphone, con hipótesis sobre la evolución de la usabilidad, la forma y las plataformas de acceso que las tecnologías de sustitución podrán traer. Esta prospectiva sobre el futuro de las comunicaciones móviles tiene en cuenta algunas de las tecnologías ya disponibles o emergentes como los sistemas de Inteligencia Artificial, las redes 5G, la impresión 3D, los sistemas de reconocimiento de voz o los nuevos materiales.

\section{Palabras clave}

Aplicaciones de mensajería, evolución funcional, interacciones sociales móviles, ecosistema móvil, plataformas anidadas.

\section{Abstract}

A decade after the launch of Apple's iPhone, smartphones have become the basic and central device of personal communication and access to the network. However, the signs of maturity that are beginning to appear in the main markets and the fact that technological evolution continues, it indicates that smartphones will be replaced by some device whose form and functions we do not know yet. In this article we analyze the possible scenarios of a post-smartphone stage, with hypotheses about the evolution of usability, the form and access platforms that substitution technologies could bring. This prospective on the future of mobile communications takes into account some of the technologies already available or emerging ones such as Artificial Intelligence systems, 5G networks, 3D printing, speech recognition systems or new materials.

\section{Keywords}

Messaging Applications, Functional Evolution, Mobile Mediated Interactions, Mobile Ecosystem, Nested Platforms.

\section{Sumario}

1. Introducción. 2. Evolución tecnológica y escenarios post-smartphone. 2.1. Escenario 1: smartphone dispositivo básico. 2.2. Escenario 2: aparición de tecnología superadora y smartphone como nicho. 2.3. Escenario 3: redes móviles dejan de ser soporte de comunicaciones personales. 2.4. Escenario 4: Computación ubicua en la nube: la desmaterialización del smartphone. 3. Conclusiones. 4. Bibliografía. 


\section{Introducción}

La telefonía móvil se puede considerar ya como el servicio más difundido y exitoso de la historia de las telecomunicaciones (Downing,1995:289) (Ahonen,2012) (GSMA, 2018), con la paradoja de que empezó siendo una propuesta de las operadoras para el cliente corporativo, sin que éstas contemplaran siquiera la posibilidad de que llegase a ser un sustituto de la telefonía convencional sobre redes fijas.

En un corto espacio de tiempo la evolución de los sistemas móviles fue dando paso a un incremento del ancho de banda disponible para el usuario, que a su vez demandó nuevos terminales con mayor capacidad de procesamiento.

Las redes europeas $2 \mathrm{G}$ fueron diseñadas teniendo en cuenta las necesidades de un usuario de telefonía móvil que todavía contemplaba ésta como un complemento del teléfono fijo de su hogar o trabajo. Es decir, una comunicación que cubría los vacíos espacio-temporales de la telefonía sobre cable.

A pesar del enorme éxito de esta segunda generación de redes móviles, la transición al $3 G$ no fue ni uniforme en todas las regiones, ni contó con un único estándar global, ni tampoco fue inicialmente aceptada como un sistema de banda ancha alternativo de acceso a Internet.

Las principales operadoras mundiales idearon una estrategia $3 \mathrm{G}$ que efectivamente conducía al acceso pleno a Internet, pero desde luego ni a corto ni a medio plazo, ya que una aceleración no controlada en los tiempos de transición se consideraba que podría poner en riesgo las inversiones en la mejora de las redes de telefonía convencional.

Esta es la razón por la cual transcurrieron seis largos (en términos tecnológicos) años desde las primeras concesiones de redes $3 G$ en Europa y Japón justo a principios de este siglo y la aparición de un dispositivo que recogiese y sobre todo, reflejase los deseos de la primera generación de usuarios de telefonía móvil de la historia.

El iPhone de Apple vino a romper el equilibrio, deseado y sostenido por la industria, entre el mercado de consumo de las computadoras portátiles y los PC de escritorio, entre los dispositivos móviles corporativos y los domésticos y finalmente, entre las redes de banda ancha fijas y móviles.

Aunque el primer iPhone de Apple, presentado en enero del 2007, no era un dispositivo técnicamente más avanzado que los Keitai japoneses de DoCoMo o los robustos móviles europeos comercializados por Nokia y Ericcsson, se convirtió en el primer smartphone que dio forma a esta categoría premium de teléfonos móviles.

La estrategia de Apple de transformar los teléfonos móviles en computadoras verdaderamente portátiles tampoco fue original, ya que la canadiense Blackberry había creado con éxito esta categoría de smartphones corporativos siete años antes.

La mayor aportación del iPhone de Apple fue precisamente realizar una convergencia de facto entre los hasta entonces teléfonos para el sector profesional y los que usaba el público en general.

Cuando los usuarios del novedoso terminal de Apple comenzaron a llevarlo a sus trabajos, se puso en evidencia la enorme diferencia entre la usabilidad amable e intuitiva del iPhone y los opacos protocolos corporativos diseñados para respetar las leyes de sus firewall.

No obstante, habría que esperar un año más (julio del 2008) para que Apple terminara de completar la oferta holística de sus iPhone, con la presentación de la tienda de aplicaciones (app store), que supuso la segunda gran aportación de Apple al mercado de la telefonía móvil.

Las aplicaciones son pequeños programas que el usuario instala en su dispositivo para realizar todo tipo de tareas tanto online como offline. Al hacer esto el usuario personaliza cada terminal, transformando el móvil en un objeto que representa su perfil único de usuario, desde sus aficiones a sus intereses culturales y profesionales.

A partir de entonces el modelo imperante en la industria fue este binomio que aúna la venta del dispositivo (hardware) con la tienda de aplicaciones (software) cuya oferta se expande y adapta conforme mejoran las características de los móviles en cada generación.

Los smartphones a partir del iPhone progresivamente fueron abandonando la categoría de teléfonos móviles para convertirse en eslabón privilegiado de la evolución de la computación personal.

Ningún analista de la industria pudo prever en la pasada década que los smartphones se convertirían en los dispositivos tecnológicos más vendidos y utilizados, sobrepasando no solamente a los PC convencionales, sino a los propios teléfonos móviles básicos.

La base instalada de smartphones (véase gráfico 1) se convirtió en un nuevo parámetro para evaluar el progreso tecnológico en este siglo, ya que no sólo representa la opción preferente para el acceso a Internet, sino la primera computadora para muchos usuarios en países en desarrollo donde no hubo una previa adopción de PC (Napoli y Obar, 2013; Comin, 2014).

Al transformarse en una computadora, los smartphones comenzaron a seguir los dictados de la ley de Moore (1965) que no es un principio científico pero actúa como el indicador básico dentro de la industria de los semiconductores, para ofertar cada 18 meses procesadores dos veces más potentes, con el consiguiente efecto de abaratamiento para los usuarios.

Con smartphones en un amplio rango de precios desde menos de 100 dólares hasta los 1000 de algunos de los últimos modelos altos de gama, sistemas operativos sobre software no propietario y sistemas de financiación gestionados por las propias operadoras, para la gran mayoría de usuarios de móviles las barreras de entrada para optar a estos dispositivos son cada vez menores (Unesco, 2016). 


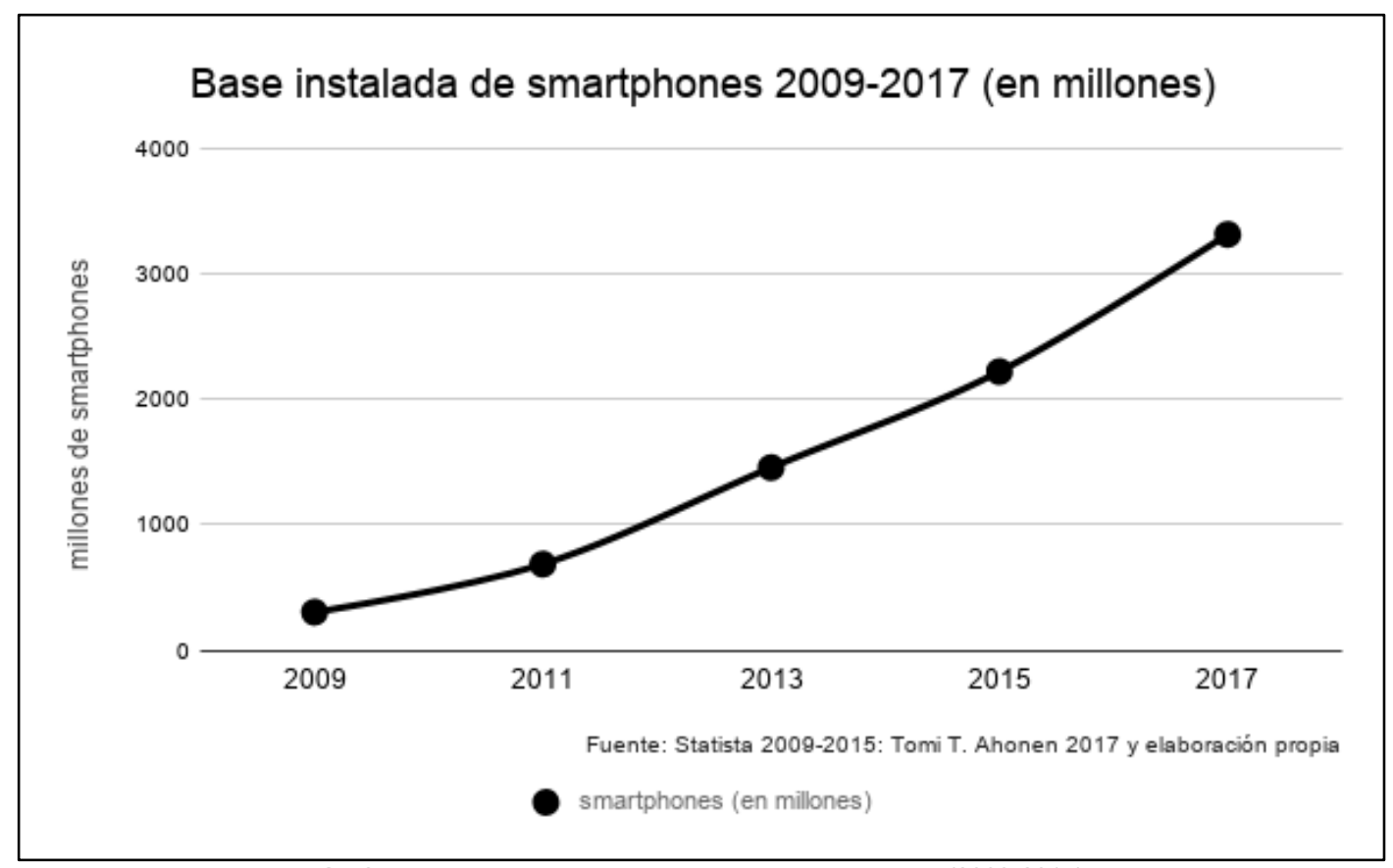

Gráfico 1: Base instalada de smartphones en el mundo (2009-2017)

La tendencia global por tanto conduce a la equiparación entre usuarios de móviles básicos y smartphones, ya que los datos muestran que el $80 \%$ de los móviles nuevos que se venden son smartphones (Ahonen, 2018) y además la tecnología que introducen extiende el ciclo de vida útil del aparato, lo que a su vez repercute en el crecimiento del mercado de segunda mano (Counterpoint, 2018) y en la base instalada de smartphones en todo el mundo.

La centralidad del smartphone como dispositivo para las comunicaciones personales, el ocio online y un creciente número de aplicaciones profesionales han convertido a este dispositivo en algo demasiado importante, cuya evolución influye en sectores clave.

La etapa pos-smartphone es compleja de formular por que "nuestra vida actual es difícil de imaginar sin él" (Shirky, 2015), a pesar de ello y asumiendo su carácter inevitable, se describen cuatro escenarios de innovación basados en la taxonomía de Freeman y Pérez sobre cambios de sistema tecnológico (88: 45) que abarca desde su mejora incremental hasta su sustitución por otro completamente diferente en el siguiente ciclo de la evolución tecnológica.

\section{Evolución tecnológica y escenarios post-smartphone}

Aunque no hay una interpretación única de cuando se inició el actual ciclo de evolución tecnológica, no obstante, si se existen hitos que sirven para explicar e identificar la posición que ocupa el smartphone dentro de ésta (véase gráfico 2).

La evolución de los smartphones es en sí misma uno de los ejemplos más claros de la convergencia como fenómeno característico de la actual fase tecnológica (Deuze, 2007: 70; Solis, 2012).

La incorporación progresiva de elementos que hasta entonces se empleaban sólo en las computadoras personales (procesadores, teclados qwerty, pantallas táctiles y de mayor diámetro), junto a la integración de programas de software específicos para estos dispositivos, convirtieron a los smartphones en una alternativa factible a los PC.

Nuevamente, ni para fabricantes de computadoras personales ni para las empresas que comercializaban teléfonos móviles fue fácil prever que la convergencia entre computadoras y los servicios de comunicaciones personales en Internet se iba a producir a partir de los móviles y no de las computadoras portátiles (notebook).

El iPhone de Apple no realizó sólo esta convergencia, pero si le dio forma, de tal modo que desde su lanzamiento comercial en junio del 2007 no se ha producido ninguna innovación relevante, tan solo un cúmulo de novedades y mejoras sobre el original. 


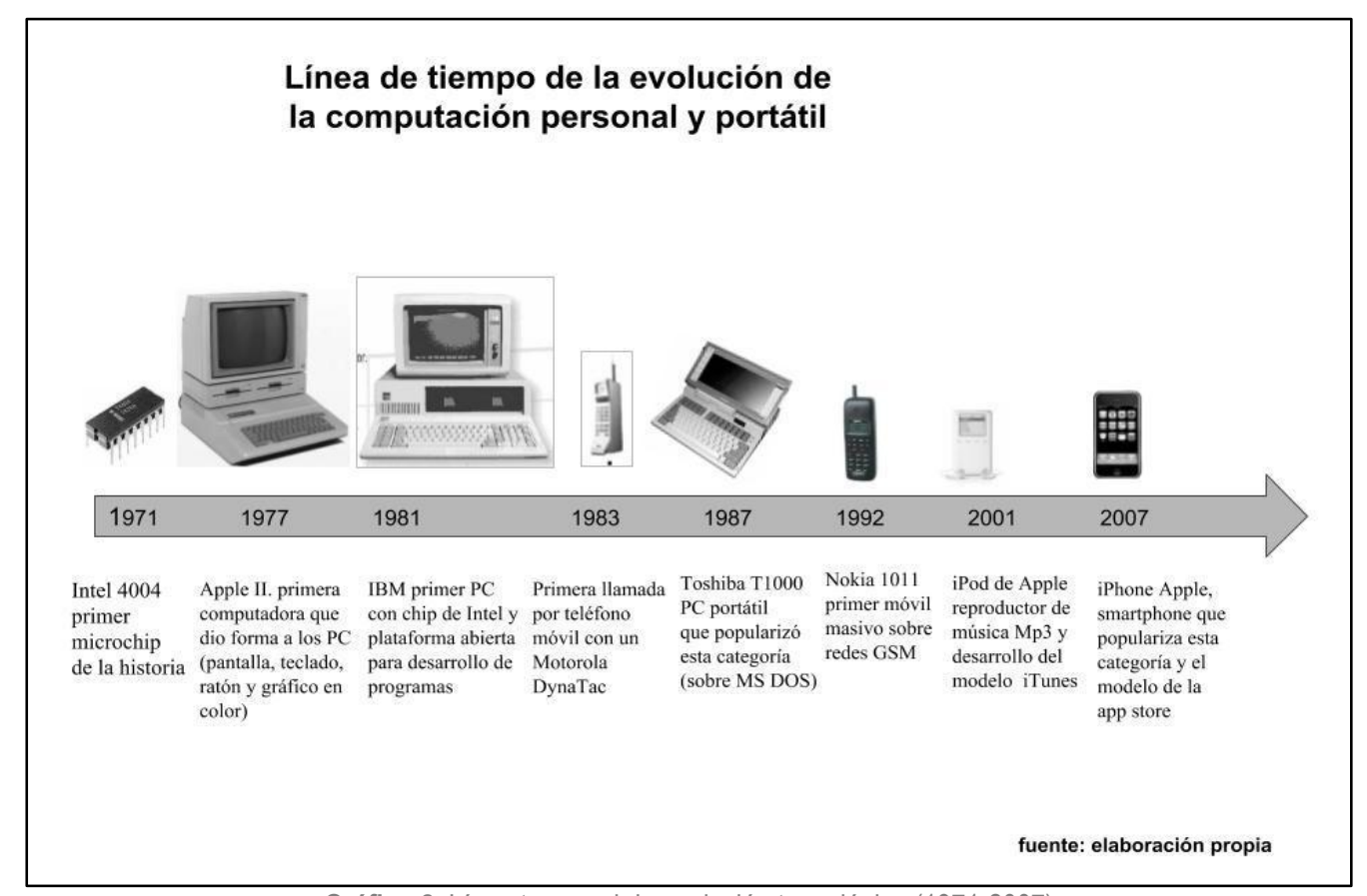

Gráfico 2: Línea temporal de evolución tecnológica (1971-2007)

La sustitución de los PC por el smartphone como dispositivo principal de acceso a Internet, convirtió a los primeros en una categoría nicho dentro del mercado tecnológico y al segundo en el principal receptor y catalizador de los avances en factores básicos como la velocidad de procesamiento, resolución de pantallas, ergonomía, duración de las baterías y desarrollo de sistemas operativos.

A su vez el smartphone marco la transición entre la primera y la segunda gran fase de Internet, el paso de las redes fijas a las redes móviles. Lo que implicó más que una sustitución tecnológica del tipo de redes, una completa redefinición de la oferta y de las formas y hábitos de consumo.

El acceso a los servicios más populares que presta la red actualmente se realiza mayoritariamente a través del smartphone, desde las redes sociales a los buscadores, pasando por la mensajería instantánea y el comercio electrónico.

Este cambio en el modo y dispositivo de acceso obligó a su vez a las empresas detrás de estos servicios básicos a rediseñar sus formatos de presentación, cambiando los lenguajes de programación y sobre todo, la selección y ordenación de elementos que se muestran.

La adopción del móvil primero (mobile first) se convirtió más que una estrategia en un imperativo de cualquier organización que deseará tener visibilidad en la red, tras una larga etapa cuando la sede electrónica principal fue la página web.

A su vez, el uso masivo de los smartphones como primer dispositivo realmente portátil abrió la posibilidad a nuevos modelos de negocio en Internet, basados en parámetros inéditos hasta entonces como la ubicación geográfica y la inmediatez en las respuestas.

Es indudable que empresas como Uber o Airbnb, dos de las más representativas de la llamada economía colaborativa (Khare, Stewart y Schatz, 2016: 129) no podrían haber alcanzado su dimensión actual sin los altos niveles de penetración del smartphone en sus principales mercados.

La popularización de los smartphones a partir del 2008 no fue razón suficiente para el cambio definitivo hacia una Internet móvil, pero si fue el factor más determinante, ya que traspasó el peso de la industria de las operadoras a los fabricantes y desarrolladores independientes de software.

A partir de aquí cualquier escenario post-smartphone implica una alteración del sistema tecnológico ya que "es un cambio de amplio alcance en tecnología que afecta distintos sectores de la economía, a la vez que propicia la aparición de nuevos sectores" (Freeman y Perez, 1988: 46).

La hipótesis propuesta incluye 4 escenarios post-smartphone que serían:

$1^{\circ}$ Smartphone como dispositivo básico (100\% penetración).

$2^{\circ}$ Aparición de tecnología superadora y smartphone como nicho.

$3^{\circ}$ Redes móviles dejan de ser soporte principal de comunicaciones personales.

$4^{\circ}$ Computación ubicua en la nube y desaparición del smartphone.

La ordenación de estos escenarios sigue una línea de evolución desde la ubicuidad del smartphone hasta su completa desaparición por sistemas integrados en la nube. A continuación, se describe cada uno de ellos.

\subsection{Escenario 1. Smartphone como dispositivo básico}

Este escenario supone aceptar que los smartphones van a sustituir por completo a los móviles básicos. de modo que la propia denominación de smartphone se vaciará de sentido. 
Con una penetración actual superior al $66 \%$ de todos los usuarios mundiales de móviles, una tasa media de incremento de ventas sobre el total de móviles del 5\% anual y teniendo en cuenta la ralentización de las ventas mundiales, que pasaron en el periodo 2014-2017 del 13\% al 1,8\%, la base instalada de smartphones alcanzaría el 100\% en 5 años (Ahonen, 2018).

Un smartphone que alcanzara la saturación no significa que llegará a toda la población del planeta, sino que el dispositivo básico que tendrían todos los usuarios de móviles seria al menos un smartphone. Para entender esta diferencia, en el año 2017 el $67 \%$ de la población mundial era usuario de móvil pero solo un 44\% lo era de smartphone (UIT, 2017; Ahonen, 2017).

Una ubicuidad absoluta del smartphone significa completar el objetivo que los líderes de la industria tecnológica denominan "los próximos mil millones" (Sengupta, 2018). En realidad, una forma unificada de referirse a los siguientes pasos estratégicos para incluir o captar 1000 millones de nuevos usuarios a Internet, fundamentalmente a través de dispositivos móviles y sobre todo, en Asia y Africa (Filloux, 2018).

Tomando como ejemplo el caso de países como los EEUU o la región europea, donde la penetración del smartphone es mayor y por tanto, más cerca están de la saturación, se pueden extraer algunas lecciones de este escenario.

Allí donde es difícil vender más smartphones a nuevos usuarios, comienzan a aparecer propuestas que si bien no son superadoras de este dispositivo móvil si apuestan por un menor uso sobre todo en los hogares.

Los altavoces inteligentes serían un ejemplo de una nueva tecnología que aunque tiene complementariedad con el smartphone a través de sus asistentes digitales de voz, supone una alternativa en usos concretos como llamadas personales, comercio electrónico y ocio doméstico (reproducción de música bajo demanda).

Bajo el punto de vista de los fabricantes de smartphones, un escenario donde todos los móviles fueran smartphones aceleraría aun más la tendencia a una concentración que ya resulta evidente, no tanto en el volumen de ventas pero si en el vital dato de los beneficios, teniendo en cuenta que Apple y Samsung suman entre las dos el 96\% de los beneficios del sector (Counterpoint, 2018; IBD, 2018).

Un escenario como el descrito de polarización del mercado conduciría a una oferta por arriba de smartphones con precios inasequibles para la mayoría y por abajo a una aparente diversidad de dispositivos, que si bien cumplen la función básica de acceso a Internet no podrían incorporar, al menos en poco tiempo, las últimas novedades tecnológicas.

Sin beneficios para la mayoría de los fabricantes, muchos llevarían sus futuras inversiones hacia otros mercados más lucrativos, reduciendo a un más la oferta comercial de smartphones.

A modo de resumen, la madurez absoluta de este mercado llevaría necesariamente a una redefinición de la categoría smartphone, algo que podría producirse vía regulación estatal o por un acuerdo dentro de la propia industria.

Fijar las características básicas de un smartphone para ser considerado como tal, aunque obligaría a una revisión periódica por los avances que se producen, generaría un segmento amplio donde muchas marcas podrían competir y abriría el camino hacia el escenario siguiente.

\subsection{Escenario 2. Aparición de tecnología superadora y smartphone como nicho}

Este escenario es el primero que contempla la vida sin smartphones como dispositivo mayoritario, ya que supone la aparición de nueva tecnología que da lugar a otros dispositivos con usos completamente diferentes a los actuales.

Para que se produzca este relevo la nueva tecnología no sólo debe ofrecer una mejora incremental sino superadora de la (actual) experiencia smartphone. Lo que relegaría a los smartphones a la categoría de nicho, es decir rentable para pocos fabricantes y no dirigida al mercado masivo. La cuestión básica sería por tanto identificar que tecnología lograría esta sustitución, algo que conduce a dos caminos: analizar una potencial evolución de tecnologías que no siendo mayoritarias están presentes actualmente, este es el caso de la realidad virtual (VR), la realidad aumentada (AR), la impresión 3D, los robots y nuevos materiales que permiten fabricar pantallas flexibles. La segunda opción nos llevaría a bordear las fronteras de la ciencia ficción, con tecnologías que aun no conocemos o de las que apenas empezamos a intuir sus usos y formas futuras como la computación cuántica.

Eligiendo para este análisis la primera opción vemos como las formas de acceso que añaden algún componente de realidad paralela o mixta, comienzan su camino ascendente dentro del "ciclo de altas expectativas" (Gartner, 2017) que representa la evolución de la madurez y el potencial de una tecnología.

Realidad virtual (VR) y Aumentada (AR) son dos desarrollos tecnológicos que llevan varios años como una posibilidad, contemplándose ambos como una forma alternativa de acceso a Internet y con una amplia clase de contenidos que incluyen desde los juegos o la narrativa de ficción a herramientas de identificación en labores profesionales.

Sin dejarse influir por los vaivenes coyunturales de determinados hitos puntuales como el éxito de Niantic con Pokemon Go en el 2016 y que convirtió la AR en una alternativa de ocio global, lo cierto es que con excepción de los videojuegos, la AR permanece aletargada como opción.

Tanto la VR como la AR tienen la ventaja de que integran el smartphone en su uso, lo que supone de partida una potencialidad, ya que los usuarios podrían seguir utilizando el mismo dispositivo.

El caso de la VR es algo más complejo, ya que aunque en efecto existen en el mercado dispositivos asequibles que permiten integrar el smartphone, otros requieren un dispositivo separado, sin que se pueda considerar un sustituto del smartphone para los usos más comunes (mensajería, llamadas, geolocalización etc.) 


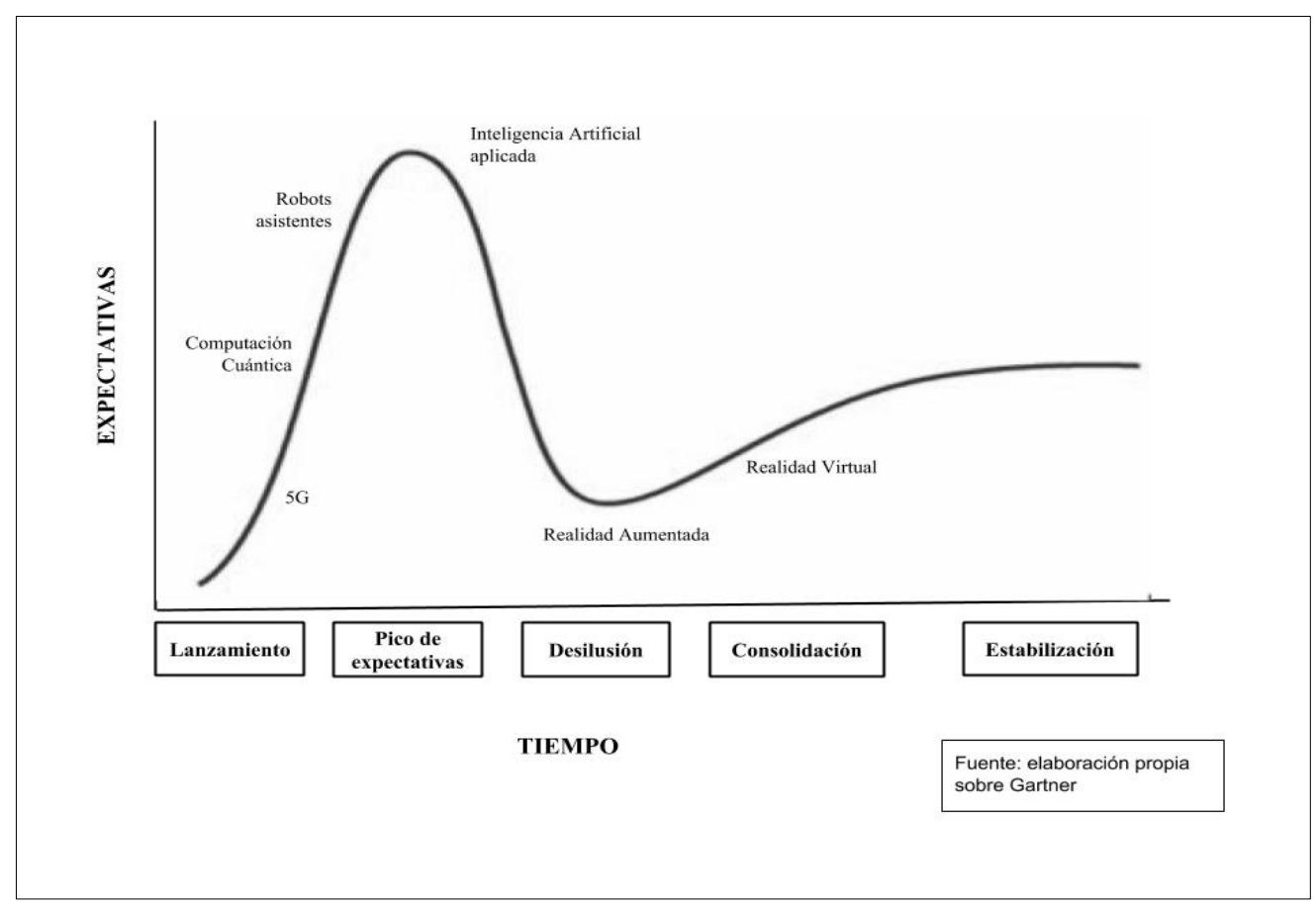

Gráfico 3: Ciclo de altas expectativas

La impresión 3D es otra de las opciones tecnológicas disponible con una creciente difusión y aunque no está relacionada directamente con las comunicaciones personales, puede tener un impacto a medio plazo en el mercado smartphone.

Unas impresoras 3D cada vez más precisas, baratas y fáciles de utilizar por un usuario común podrían conducir a una fabricación a la carta y personalizada de muchos componentes, sobre todos externos, de los smartphones.

Este cambio en los hábitos de consumo, expulsaría del mercado a muchos fabricantes ya que para una parte de los usuarios el smartphone sería básicamente la placa con la circuitería y el sistema operativo, o sea un modelo que recuerda bastante a la primera etapa de la computación personal "con PC's que invitaban a que otros innovasen" (Zittrain, 2008: 3).

Este escenario como vemos no prescinde del smartphone, pero si altera por completo las reglas del sector, incrementando solamente los ingresos de aquellos fabricantes como Apple que lograron convertir sus dispositivos en fetiches culturales.

Una tercera opción en este escenario serían los wearables, definidos como "una categoría de dispositivos que el usuario puede llevar encima y que recopilan activa o pasivamente datos" (Davidson, 2016).

Dentro de estos se encuentran algunos dispositivos populares aunque todavía sin una difusión masiva y que utilizan el prefijo smart para diferenciarse de los modelos previos analógicos o sin conexión. En esta categoría podemos ver desde relojes, pulseras biométricas, gafas, anillos hasta ropa y calzado.

La "computación wearable" tal como la definió Steve Mann hace 21 años (Mann, 1997) se contempla no solo como una nueva aproximación a la computación, sino como la siguiente etapa en la evolución tecnológica. (véase gráfico 4)

Algunos dispositivos wearables, sobre todo los relojes y las pulseras, prometen sustituir por completo al smartphone, al menos cuando el usuario se desplaza, ya que las últimas versiones contienen conexiones que permiten acceder directamente a redes móviles (3G/4G) y wifi.

La principal barrera para su adopción hasta ahora ha sido el limitado diámetro de pantalla, un argumento no obstante que recuerda las primeras críticas al hábito de consumo en los móviles y que por tanto, supone una incógnita respecto a que consideración le darán los "adaptadores tempranos"(Moore, 2015: 31) y sobre todo, las generaciones más jóvenes educadas a partir del smartphone.

Con unos wearables como dispositivo preferente en movilidad, el smartphone sufriría un desplazamiento hacia su consumo en los hogares, una tendencia largamente detectada en muchos estudios sobre el consumo en móviles (PewInternet, 2018; OfCom, 2018; EGM, 2018).

Unos wearables considerados lo suficientemente eficaces para funciones como mensajería, llamadas de voz, consumo troceado de contenidos de entretenimiento o información y que además proporcionen controles de variables vitales de los usuarios, podrían lograr una transición no abrupta hacia una reducción del uso de smartphones, es decir la esencia de este segundo escenario. 


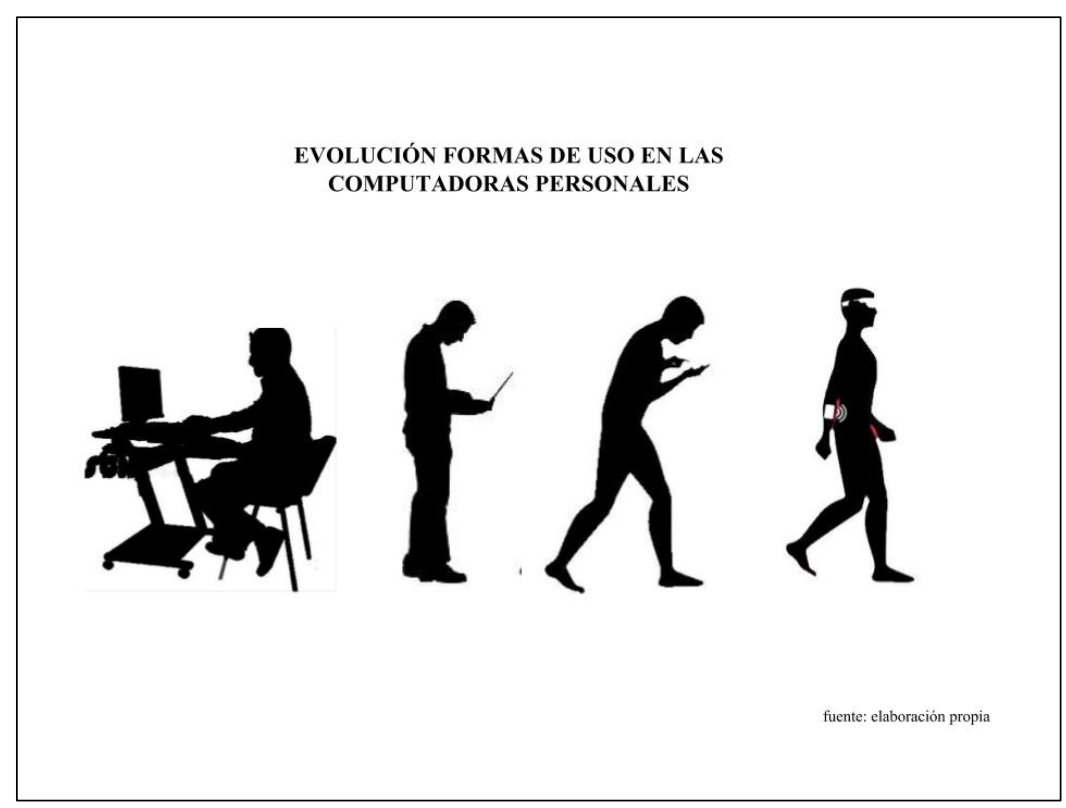

Gráfico 4: Evolución formas de uso

\subsection{Escenario 3. Redes móviles dejan de ser soporte de comunicaciones personales}

La evolución de los sistemas digitales de telefonía móvil ha ido describiendo una paradoja histórica: cada vez se usan menos para llamadas vocales y cada vez más para tráfico de datos, es decir un uso que hasta hace poco se reservaba para las comunicaciones corporativas.

La propia evolución del término teléfono móvil en favor del más simple concepto de móvil ilustra suficientemente el cambio de uso de dispositivo para llamadas a uno que permite el acceso a servicios en Internet.

Por otra parte, la misma evolución de los sistemas digitales de telefonía móvil $(2 \mathrm{G} / 3 \mathrm{G} / 4 \mathrm{G})$ explica en parte este cambio de uso preferente, ya que cada uno añadió mayor ancho de banda disponible, lo que supuso disponer de una capacidad sobrante, ya que las llamadas de voz incluso incrementando su calidad ocupan un espacio muy limitado.

Este cambio supuso un impacto directo en la transformación de los modelos de negocio de las operadoras de telecomunicaciones, convirtiéndose en proveedores de acceso a Internet.

A partir de este transcendental cambio los precios de las comunicaciones personales comenzaron a describir la drástica reducción de precios que caracteriza todo modelo económico basado en bits (Kelly, 1998).

En este escenario por tanto, las condiciones económicas del mercado de la banda ancha determinan un cambio de objetivos de las antiguas operadoras, de las comunicaciones entre personas a la comunicación entre objetos o Internet de las Cosas (loT).

Si las comunicaciones personales dejan de ser rentables para las operadoras, esto no significa que desaparezcan sino que se canalizarán a través de otras redes, con diferentes modelos de financiación. Por ejemplo wifi públicas o privadas o como parte indistinguible del paquete de datos contratado por un usuario.

Un smartphone liberado de su pasado telefónico tendría un impacto directo en su diseño y en los componentes que incluye (¿tarjeta SIM?), lo que a su vez lo acercaría aún más a un terminal manual de acceso a la red.

Este cambio no seguiría necesariamente la tendencia a la convergencia de dispositivos que vimos en la primera etapa (2007-2018), sino que abriría al menos tres alternativas:

- dispositivos telefónicos para hablar y mensajería básica, sin acceso a Internet o con uno muy básico.

- computadoras móviles con o sin pantalla, que básicamente actúan como procesador y router de conexión con periféricos portátiles inteligentes (auriculares, gafas conectadas)

- $\quad$ smartphones evolucionados, pero basados en los actuales, con cada vez más prestaciones, componentes de mayor calidad, con ciclos de sustitución cada vez más extensos, precios altos y mayor margen de beneficio para el fabricante.

Este despliegue de la oferta sitúa al smartphone, tal como se conoce ahora, como una opción minoritaria pero lucrativa en el segmento de los dispositivos personales, que extendería su ciclo de vida hasta el siguiente escenario.

\subsection{Escenario 4. Computación ubicua en la nube: la desmaterialización del smartphone}

La digitalización no es sólo un proceso de sustitución de sistemas analógicos por digitales, fundamentalmente representa una tendencia a transformar en programas de software casi cualquier dispositivo, algo que obedece al "nuevo imperativo" que identificaron autores como Negroponte (1995), por el cual todo lo que pueda ser reducido a bits finalmente lo será. 
La desmaterialización de soportes culturales y de ocio como el libro, el CD y el DVD, fue sin duda la primera de las grandes innovaciones de la primera etapa de Internet, cuando el PC conectado a una línea fija era una metáfora misma de esta red.

Pero esta ola digitalizadora prosiguió su lógica de transformación convirtiendo en bits a los propios dispositivos, algo que redujo exponencialmente su valor. Este proceso solo se puede entender atendiendo al extraordinario desarrollo de los "recursos de computación compartidos que llamamos la nube" (Rayes y Salam, 2017).

La nube es una metáfora en si misma ya que permite imaginar la red como un catálogo de recursos bajo demanda, donde tiene más sentido el acceso que la posesión.

Dentro de esta tendencia, el smartphone proporcionó un primer ejemplo de cómo dispositivos de uso común como las cámaras, las videoconsolas o los mismos PC's se transformaron en aplicaciones o pequeños programas para descargar.

No existe por tanto ninguna razón para no pensar en la hipótesis de un smartphone transformado a su vez en aplicación o en otras palabras, accesible desde que cualquier dispositivo conectado.

La portabilidad del smartphone sería sustituida por la ubicuidad de la nube que se extendería hasta donde alcance la propia cobertura de las redes. Y el smartphone pasaría a definirse como un conjunto de funciones y no de los elementos (pantalla, unidad de memoria, procesador etc) que conforman actualmente este dispositivo.

Un smartphone en la nube desmaterializado ahondaría aún más en la personalización como factor esencial en el acceso. Esto significa que en función del uso individual se puede asignar más o menos recursos, tales como capacidad de procesamiento, memoria o velocidad de acceso.

La tendencia a la personalización de los smartphones fue de hecho uno de los efectos de la popularización de las tiendas de aplicaciones, a partir del modelo de la app store de Apple en el 2008.

Si las aplicaciones personalizaron el software instalado en el dispositivo, las plataformas en la nube harían lo mismo con el hardware, creando un recurso tecnológico único para cada usuario, ajeno por completo a cualquier ciclo de obsolescencia.

Este escenario de radical evolución no obstante ofrece distintas interpretaciones que a su vez se corresponden con fases de implantación.

- $\quad$ Fase 1: los smartphones crean réplicas digitales (digital twins) en la nube, de modo que cada dispositivo de hardware en manos de un usuario puede ser controlado desde la red y ofrecer información sobre su estado y compatibilidad con otros dispositivos y sistemas.

- Fase 2: el smartphone se convierte en un conjunto de herramientas configurables en la nube, permitiendo el acceso desde cualquier pantalla conectada con mínimos requisitos técnicos y por tanto, con un coste de adquisición declinante.

- $\quad$ Fase 3: la desmaterialización del smartphone de la fase anterior, genera un nicho de mercado para fabricar, a escala casi artesanal. dispositivos con materiales de alta calidad que representen un cierto status de usuario.

\section{Conclusiones}

Los smartphones se convirtieron en menos de un decenio en el gran factor democratizador del acceso a las telecomunicaciones y la computación de la historia.

Durante este vital periodo, la transformación del móvil básico en computadora portátil sometió a éste a procesos exponenciales de mejora de su rendimiento, conocidos en ciclos anteriores, que a su vez redujeron su precio logrando extender su franja de usuarios hasta los segmentos de menor poder adquisitivo.

El éxito, sin apenas precedentes, de su difusión temporal produjo una reorientación de las inversiones en la industria tecnológica, ante la evidencia de que para la mayoría de los usuarios no solo se trataba de una mejora respecto a los teléfonos móviles anteriores, sino un sustituto de la computadora personal, sobre todo para el usuario no corporativo.

En la actualidad, las ventas de móviles representan el 24\% de los ingresos totales del sector (Ahonen, 2016), con una acusada tendencia hacia la concentración de ingresos en oligopolio.

Como consecuencia Apple, líder por ingresos y beneficios del sector, se ha convertido en la empresa de mayor capitalización bursátil del mundo (Forbes, 2017), un hecho inédito pero enmarcado a su vez en la tendencia hacia una mayor valoración de las compañías tecnológicas en detrimento de las financieras y de explotación de hidrocarburos.

La dimensión y proyección en el mercado mundial de las empresas que conforman el sector smartphone acrecienta la necesidad y transcendencia de una prospectiva sobre la siguiente fase de evolución tecnológica o post-smartphone.

Los escenarios analizados en este articulo demuestran que a corto y medio plazo se va producir una continuidad en la adopción del smartphone, pero a una velocidad mucho menor que a principios de la década actual, debido a la madurez de los principales mercados. 
La adopción universal del smartphone como dispositivo principal de acceso a la red, dejará sin sentido la propia denominación de esta categoría de dispositivos y abrirá una nueva fase que podría concluir con su desaparición, al transformarse en un conjunto de funcionalidades personalizadas en la nube.

Una nube que provea estas funciones básicas y avanzadas de comunicación y computación dejaría un margen muy estrecho, pero lucrativo, para un número muy reducido de fabricantes con ciclos de renovación más extensos que liberarían a estas empresas de la presión por ofrecer novedades anualmente y les permitiría concentrarse en innovaciones de verdadero valor añadido para el usuario.

El desarrollo e implantación de sistemas de Inteligencia Artificial tendrá un doble impacto tanto en el análisis de las verdaderas necesidades de cada usuario, como en una optimización de toda la cadena de valor de la industria.

La adopción del 5G, como siguiente generación de sistemas de telefonía móvil, tendrá consecuencias directas en el diseño de los smartphones, en su uso, pero también en los modelos de negocio de las operadoras.

Todas las generaciones anteriores, desde el $2 \mathrm{G}$ al $4 \mathrm{G}$, han descubierto formas de uso inéditas por parte de los usuarios, y también cambiaron la composición de los ingresos de las operadoras de telecomunicaciones. Durante este periodo, que abarca la última década del siglo XX y las dos primeras de este siglo, las operadoras pasaron de ingresos basados en redes fijas a móviles y de llamadas a datos.

Un escenario post-smartphone supondría un impacto global, pero de distinto nivel en cada región. Así, en Asia y los países emergentes de la cuenca del Pacifico, el impacto será alto ya que allí se localizan las mayores cadenas de fabricación de componentes y ensamblaje, todavía con una fuerte participación de mano de obra intensiva a pesar de los evidentes avances hacia la automatización (Nikkei, 2018).

En Europa el impacto sería moderado por que los fabricantes apenas representan 8\% del valor total del sector (GSMA, 2018b: 21), pero las operadoras, que generan el $62 \%$ de todos los ingresos, se verán obligadas a cambiar sus modelos de oferta lo que tendrá un impacto a medio plazo sobre sus cuentas y la cantidad y composición del empleo.

En Norteamérica, la región con mayor penetración del smartphone mundo (GSMA, 2018a: 15), sede de las empresas líderes en hardware y software del sector y la primera que marcó la transición de los PC a los smartphones, la evolución hacia los escenarios post-smartphone supondrá una reconfiguración completa del sector IT y una nueva reorientación del capital riesgo que financia el I+D de sus centros tecnológicos de la costa oeste (Baily, Reiss y Winston, 1998: 188).

Para finalizar y desde el punto de vista del impacto en el usuario de un escenario donde el smartphone dejara de ser el dispositivo por excelencia, los hábitos sociales derivados del uso de este dispositivo y que son un signo distintivo de la cultura actual, serán sustituidos o se transformaran siguiendo las pautas que vimos en ciclos anteriores.

Una nueva generación de usuarios empezará a considerar que el acceso en la nube a los recursos de comunicación, procesamiento y almacenamiento de información, que ahora realizan en el smartphone será tan normal como el acceso a la red eléctrica.

\section{Bibliografía}

Ahonen, T. (2012). The Mobile Moment is Only Months Away - Preparing For the Biggest Number Ever. Disponible en: https://goo.gl/SzfaeK

Ahonen, T (2016). Time For 2016 Total Mobile Numbers: The update to my most popular blog article in any year. Disponible en: https://goo.gl/k8ti6

Ahonen, T. (2017). World have 5.8 billion mobile phone handsets. Disponible en: https://goo.gl/aEwnrQ

Ahonen, T. (2018). Smartphone stats: Full Year 2017. Disponible en: https://goo.gl/2ZzWN6

Comin, D. (2014). The evolution of technology. Diffusion and the great divergence. Brookings Agosto. Disponible: https://www.brookings.edu/wpcontent/uploads/2014/08/Session-3-Leapfrogging-Comin_FINAL.pdf

Counterpoint (2018). Refurbished Handset Report. Disponible en: https://www.counterpointresearch.com/refurbished-handset-report/

Counterpoint (2018). iPhone X alone generated 35\% of the total handset industry. Disponible en: https://www.counterpointresearch.com/iphone-Xalone-generated-35-total-handset-industry-profits-q4-2017I

Davidson,S., Weberg, D., Porter-O'Grady, T. y Malloch, K. (2016). Leadership for evidence based innovation. Burlington: Jones \& Barlett Learning

Deuze, M. (2007). Media Work. Malden: Polity Press

Downing, J., Mohammadi,A. y Sreberny, A.(1995). Questioning the media. A critical Introduction. Thousand Oaks: Sage

AIMC (2018). Audiencia de Internet. Febrero/Marzo 2018. Disponible en: https://www.aimc.es/a1mc-c0nt3nt/uploads/2018/04/internet118.pdf

Baily, M., Reiss, P. y Winston, C. (1998). Economic Activity.Microeconomics. Washington: Brookings Institution 
Filloux, F. (2018). Mark Zuckerberg's long game: the nex billion. Disponible en: https://mondaynote.com/mark-zuckerbergs-long-game-the-nextbillion-af2e359e3bde

Forbes (2017). The world's biggest public companies. Disponible en: https://www.forbes.com/global2000/list/\#header:marketValue_sortreverse:true

Freeman, C. y Perez, C. (1988). "Structural crises of adjustment. Business Cycles and Investment Behaviour" en Dosi et al Technical change and Economic Theory. London: Francis Pinter

GSMA (2018a). The mobile Economy 2018. Disponible: https://www.gsma.com/mobileeconomy/wp-content/uploads/2018/02/The-MobileEconomy-Global-2018.pdf

GSMA (2018b). The mobile Economy. Europe 2017. Disponible en:

https://www.gsmaintelligence.com/research/?file=89a59299ac2f37508b252124726a1139\&download

IDB (2018). Apple rakes in $87 \%$ of smartphone profits. Disponible en: https://www.investors.com/news/technology/click/apple-rakes-in-bulk-ofsmartphone-profits-but-small-slice-of-unit-sales/

Kelly, K. (1998). New Rules for the new economy. New York: Viking

Khare, A., Stewart, B. y Schatz, R (2016). Phanton Ex Machina: Digital Disruption's role in business model transformation. Suiza: Springer

Mann, S, (1997). Wearable computing: A first step toward personal imaging. Cybersquare, Computer, 30(2) Febrero. Disponible en: http://wearcam.org/ieeecomputer/r2025.htm

Moore, G. (2015). Cruzando el abismo. Barcelona: Gestión2000.

Moore, G, (1965). Cramming more components onto integrad circuits. Electronics, 38(8). Abril. Disponible en: http://www.monolithic3d.com/uploads/6/0/5/5/6055488/gordon_moore_1965_article.pdf

Napoli, P. y Obar, J. A. (2013). Mobile leapfrogging and digital divide policy. Fordham University Schools of Business Research Papers. Disponible en: https://papers.ssrn.com/sol3/papers.cfm?abstract id $=2263800$

Nikkei (2018). Foxconn plots \$4bn automation push as labour cost bite. Disponible en: https://asia.nikkei.com/Business/AC/Foxconn-plots-4bnautomation-push-as-labor-costs-bite

OfCom (2018). Adult's media use and attitudes Report. Disponible en: https://www.cdc.gov/

PewInternet (2018). Mobile Fact Sheet. Disponible en: http://www.pewinternet.org/fact-sheet/mobile/

Rayes, A. y Samer, S. (2017). Internet of things: from hype to reality. Suiza: Springer

Sengupta, C. (2018). The nex billion users are the future of Internet. Disponible en https://www.blog.google/perspectives/caesar-sengupta/nextbillion-users-are-future-internet/

Shirky, C. (2015). Little rice, Smartphones, Xiaomi and the chinese dream. New York: Columbia Global Reports

Solis, B. (2012). The end of business as usual. Hoboken. John Wiley \& sons

UIT (2017). Measuring the Information Society Report 2017. Disponible en: https://www.itu.int/en/ITU-D/Statistics/Pages/publications/mis2017.aspx

UNESCO (2016). Science Report: Towards 2030. Disponible en: http://unesdoc.unesco.org/images/0023/002354/235406e.pdf

Zittrain, J. (2008). The future of the Internet and how to stop it. New Haven: Yale University Press.

\section{Cómo citar este artículo en bibliografías - How to cite this article in bibliographies / references:}

VACAS-AGUILAR, F. (2018): "El día después del smartphone: escenarios en la evolución de dispositivos móviles". En Revista de la Asociación Española de Investigación de la Comunicación, vol. 5, número 10, pp. 20-29. 